\title{
Development of Astable Multivibrators Powered by Photovoltaic Cells
}

\author{
E. C. Bento ${ }^{1}$ and A. C. C. Telles ${ }^{2}$ \\ ${ }^{1}$ Federal Institute of São Paulo - campus Campinas \\ ${ }^{2}$ Renato Archer Center for Information Technology \\ e-mail: \{ecbento, actelles\}@cti.gov.br
}

\begin{abstract}
1.Abstract
This article describes the simulation results of an astable multivibrator aimed to be fed by a photovoltaic cell, with the purpose of energy harvesting for electronic systems. The circuit was simulated using metal-oxide semiconductor field effect transistors (MOSFETs) and bipolar junction transistors. The use of half-wave and full-wave rectifiers for DC output voltage supply was also analyzed. The circuit reached a peak efficiency of $28 \%$ when using MOSFETs PMDXB550UNE and halfwave rectifier.
\end{abstract}

\section{Introduction}

Energy harvesting is the process of capturing and adapting ambient energy to power electronic systems. This area of knowledge is the object of intense study, as it allows autonomous systems not to need batteries or supercapacitors as power sources. Devices with the ability to draw energy from the environment sources like sunlight, vibration, heat or electromagnetic waves are being used more and more.

The capture of light energy is carried out by photovoltaic cells, normally produced with semiconductor material, being silicon the most used in these systems. Photovoltaic silicon cells typically supply voltage from $0.5 \mathrm{~V}$ to $0.6 \mathrm{~V}$ range, with the maximum power dependent on their area [1].

This work proposes an energy harvesting circuit to be powered by a photovoltaic cell with efficiency above 50 $\%$ and minimum operating voltage of $0.5 \mathrm{~V}$. The goal is to provide a minimum DC voltage of $4.0 \mathrm{~V}$ in the load, enough to supply a low dropout regulator. The configuration used is an astable multivibrator that has already been published [2]. In that work, the circuit was powered by a minimum voltage of $0.6 \mathrm{~V}$ and provided an output of $5.6 \mathrm{~V}$ with a power of $1 \mathrm{~mW}$ from a supply of $700 \mathrm{mV}$.

The performance of the circuit is evaluated by experimenting metal-oxide-semiconductor field effect transistors (MOSFETs) and bipolar junction transistors (BJTs). Half-wave and full-wave rectifiers were tested to obtain the DC voltage at the load. The circuits were analyzed using the LTSpice simulation program.

\section{Circuit with Half-wave Rectifier}

The circuit with half-wave rectifier and MOSFETs is shown in Fig. 1. L1, L2 and L3 are magnetically coupled. L3 powers the half-wave rectifier. L1 and L2 are $9 \mu \mathrm{H}$ and L3 is $780 \mu \mathrm{H}$. The turn ratio between L1 or L2 and L3 is approximately 9.6. D1 is a signal rectifier diode RRE02VS4S, which has low forward voltage. C1 is a $1 \mu \mathrm{F}$ capacitor. $\mathrm{R} 1$ represents the load of the circuit.

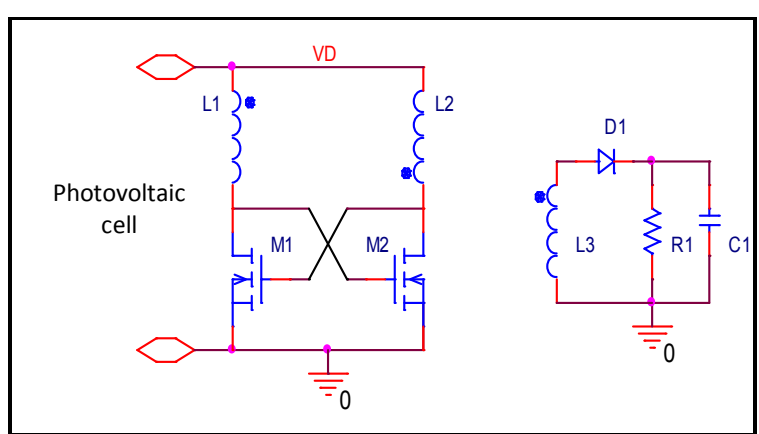

Fig 1. Oscillator using MOSFETs and half-wave rectifier

The circuit was simulated with BJTs MAT02 (named B1) and with MOSFETs BSH103 (named M1) and PMDXB550UNE (named M2).

In order to have $4.0 \mathrm{~V}$ output voltage, the circuit oscillated with a supply of $0.60 \mathrm{~V}$ and $1 \mathrm{M} \Omega$ load using $\mathrm{B} 1$; with $0.59 \mathrm{~V}$ and $3 \mathrm{k} \Omega$ using $\mathrm{M} 1$ and with $0.50 \mathrm{~V}$ and $5 \mathrm{k} \Omega$ using M2.

The graph with the circuit performance as a function of the supply voltage using the three transistors for a fixed load of $5 \mathrm{k} \Omega$ is illustrated in Fig. 2. As can be seen from this figure, M1 needs to be supplied by a higher voltage to oscillate and, therefore, to deliver power to the load. It must be said that, even with low performance, the circuit with B1 was not able to supply the minimum voltage of $4.0 \mathrm{~V}$ specified. The M2 circuit operated over the required voltage range.

\section{Circuit with Full-wave Rectifier}

The circuit with full-wave rectifier is illustrated in Fig. 3. Basically, L4 was added, magnetically coupled to the other coils and another diode RRE02VS4S. 


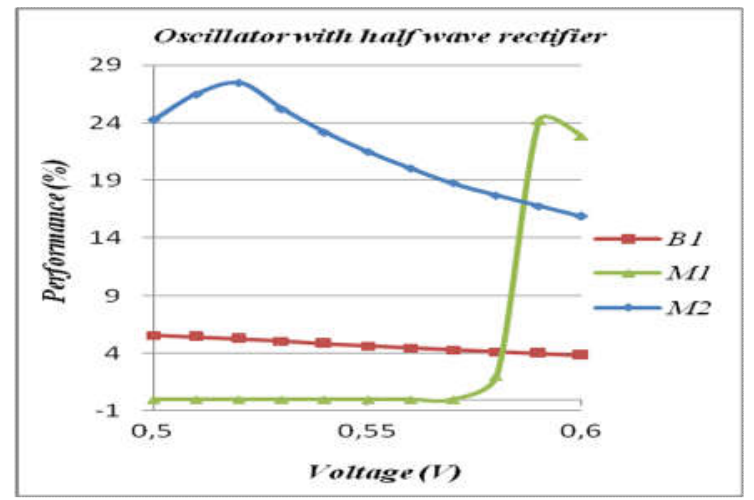

Fig 2. Performance graph for the oscillator with half-wave rectifier

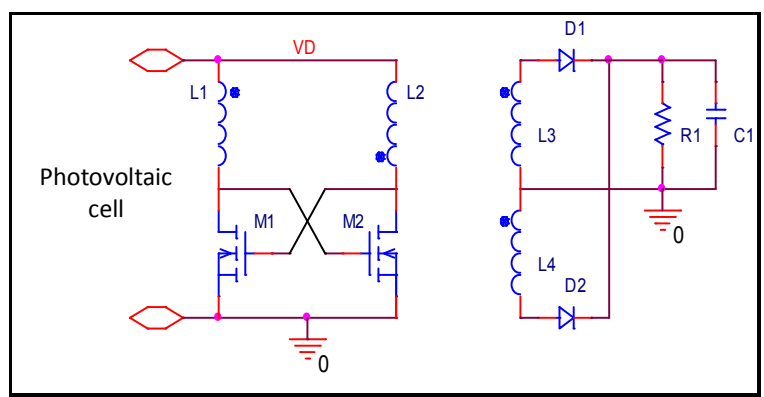

Fig 3. Oscillator using MOSFETs and full-wave rectifier

The graph with the circuit performance using the three transistors for a fixed load of $5 \mathrm{k} \Omega$ is illustrated in Fig. 4.

The circuit did not oscillate with a voltage below $0.60 \mathrm{~V}$ with any of the transistors. With $\mathrm{M} 1$, an even higher voltage, $0.62 \mathrm{~V}$, was needed so that it could oscillate with the specified load. As in the previous case, M2 operated over the required voltage range.

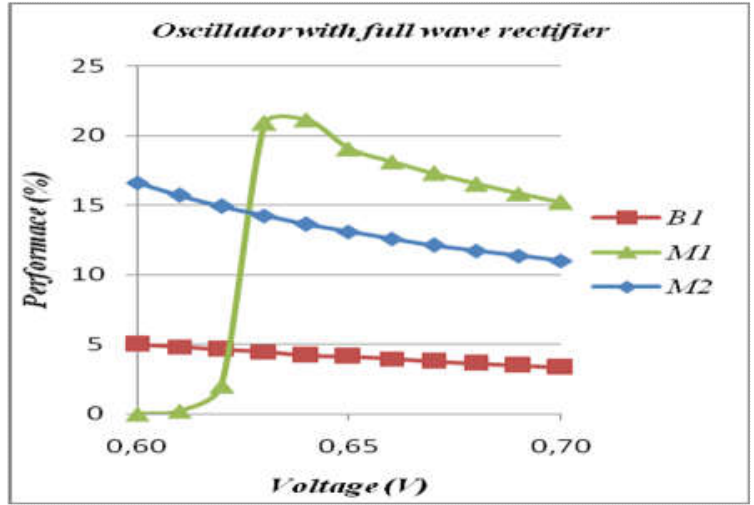

Fig 4. Performance graph for the oscillator with full-wave rectifier

\section{Impact of Vto on Performance}

Vto (parameter connected to the threshold voltage) of M2 was modified in order to observe its influence on the performance. Circuits with half-wave and full-wave rectifiers were simulated. The graph with the performance of both circuits as a function of Vto is illustrated in Fig. 5. The supply voltage is $0.50 \mathrm{~V}$ and the load resistance is $10 \mathrm{k} \Omega$. When using the full-wave rectifier, the circuit stops oscillating with Vto of $0.75 \mathrm{~V}$.

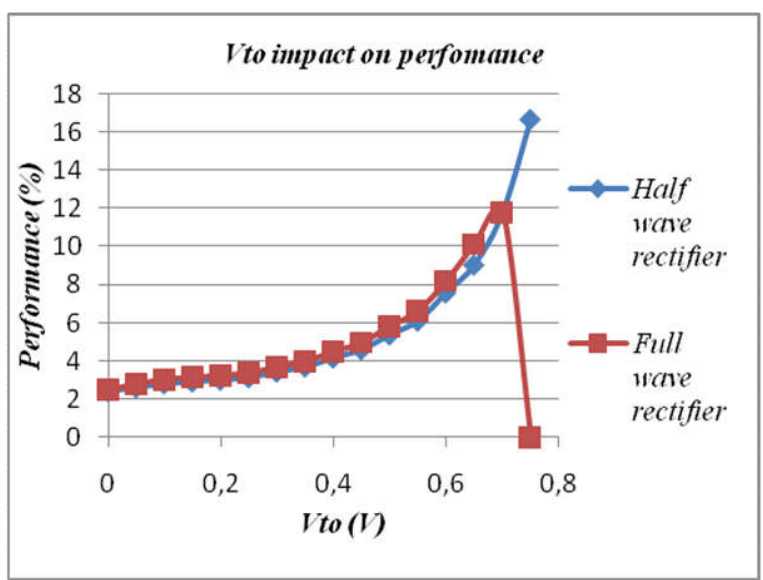

Fig 5. Performance graph as a function of Vto

\section{Conclusions}

The circuit topology presented better performance with MOSFETs instead of BJTs. Using both rectifiers, the PMDXB550UNE transistor was able to operate over the entire voltage range, which did not happen with the BSH103 transistor.

Probably the difference in operating voltage range of the circuit with a half-wave rectifier and that with a fullwave rectifier is due to the unbalanced behavior of the first. Thus, one transistor would be in charge of maintaining the oscillation and the other would supply the load in the circuit. In the case of using the full-wave rectifier, the load impacts both transistors, making a higher voltage necessary to be able to oscillate.

The best configuration found was the use of the PMDXB550UNE transistor with half-wave rectifier. Even so, the expected performance (minimum of 50\%) was not achieved.

The modification of Vto did not bring any advantage to the circuit supplying a fixed load. It is necessary to verify, however, if the circuit does not have a greater power capacity with the modification of the Vto and its performance when driving different loads.

\section{Acknowledgment}

The authors would like to thank $\mathrm{CNPq}$ by the scholarship of E. C. Bento.

\section{References}

[1] J. Carneiro, "Semicondutores - modelo matemático de célula fotovoltaica", Universidade do Minho, Portugal, 2010.

[2] A. C. C. Telles, S. Finco, and J. L. Emeri Jr., "Demonstration of a low voltage power converter with application to photovoltaic cells", In: Proceedings of X Workshop on Semiconductors and Micro \& Nano Technology (SEMINATEC 2015), 2015. 